\title{
F344-derived bone marrow cells restore injured liver with retrors ine poisoning and partial hepatectomy in analbuminemic rats
}

\author{
Biao Zhang ${ }^{1}$, Bojian Jiang ${ }^{1}$, Mitsuhiro Inagaki ${ }^{2}$, Yan Xia $^{1}$, Shinichi Kasai ${ }^{2}$ \\ ${ }^{I}$ Department of Surgery, No.3 People’s Hospital Affiliated to Shanghai Jiao Tong University, School of Medicine, Shanghai 201900, \\ China; ${ }^{2}$ Department of Surgery, Asahikawa Medical College, 2-1-1-1 Midorigaoka Higashi, Asahikawa 078-8510, Japan
}

Fischer 344 rats (F344) and congenic Nagase's analbuminemic rats (F344alb) were used as cells transplantation donor and recipient respectively. F344alb were given 2 doses of the pyrrolizidine alkaloid RS (30 mg/kg, i.p.), 2 weeks apart, followed 4 weeks later by transplantation of $1 \times 10^{7}$ BMCs (via the portal vein), freshly isolated from F344, immediately after a standard 2/3 partial hepatectomy (PH). As contrast, some F344alb administered in same conditions were transplanted $2 \times 10^{6}$ hepatocytes from F344. As the results showed that the survival rate was increased by BMCs transplantation, the percentage of regenerative live weight was increased by hepatocytes transplanted significantly. All liver function tests increased significantly in rats with only RS-exposed combined PH 2 days postPH. BMCs transplantation could protect all liver function after liver damaged by RSexposed and PH, similar result in hepatocytes transplantation except an increased AST. Clusters ( $>3$ alb+hepatocytes) were detected in livers of the recipients after hepatocytes or BMCs transplantation and the normal albumin gene sequence was detected in the liver of F344alb with hepatocytes or BMCs transplantation. Serum albumin levels were increased in recipients after BMCs transplantation and hepatocytes transplantation. Therefore, the results in present study demonstrated that F344-derived BMCs transplantation can availably advance restoration of the injury liver in F344alb, and increase the survival rate in serious liver-damaged recipients forcefully. The present investigation improved the effectiveness of BMCs transplantation, and evidential for future clinical use.

Keywords: bone marrow cell, transplantation, retrorsine, liver regeneration

Cell Research (2008) 18:s168. doi: 10.1038/cr.2008.258; published online 4 August 2008

Correspondence: Biao Zhang

E-mail: zhb6914@hotmail.com 